\title{
INTRINSIC DYNAMICS AND PHARMACOMETRIC MODEL DISCRIMINATION
}

\author{
Meyer Katzper \\ Food and Drug Administration \\ Center for Drug Evaluation and Research \\ Rockville, Maryland 20857, U.S.A.
}

\begin{abstract}
Comparison of two competing pharmacometric models is undertaken using graphic representations to elucidate their similarities and differences. The effect compartment model and the indirect response model are simulated and compared for a range of parameters to determine to what extent their dynamic characteristics can be matched. Visual tools such as hysteresis curves are used to examine model dynamics in a variety of ways. Differentiation is facilitated to the extent that competing models have distinctly defined dynamics. For the models studied here, the degree of difference depends on the region in parameter space which in turn relates to clinical response.
\end{abstract}

\section{INTRODUCTION}

Clinical trials are used to establish the safety and efficacy of drugs. Dose levels and frequency of medication are set for testing both safety and efficacy. Data from clinical trials are then studied to characterize the response to medication levels in terms of successful endpoints and adverse events. Efficacy and adverse events are both affected by the amount of drug the person is exposed to. Trials serve to estimate optimal dosages. Optimality varies by individual depending on drug concentration and drug sensitivity. The same dosage leads to different concentrations in individuals due to many causes, such as variability in rates of metabolism and drug clearance. Pharmacokinetics, therefore, focuses on determining blood plasma concentrations of drugs. Pharmacokinetics deals with the response to the presence of drugs. A central idea is the postulation of a direct link between concentration and response. This framework led to progress in many cases by guiding the adjustment of dosages to get desired concentration levels.

Taking the concentration-response viewpoint literally failed to explain cases where greater effects occurred after concentrations were falling. This paper deals with two classes of models formulated to account for this and the similarities and differences in model predictions.

\section{BACKGROUND}

Compartmental analysis assumes that the distribution of drugs in the body can be modeled by a discrete number of well stirred compartments. In the simplest case one compartment can satisfactorily fit the plasma concentration data. We deal with such a case. Effects considered are those which lag plasma concentration. We do not consider lag cases where the cause for delay of effect is the time needed for a drug in plasma to equilibrate with a target organ as such cases are accounted for by multicompartmental models.

When delayed effects are graphed versus plasma concentration, the data form loops with two values associated with a single concentration (Roland and Tozer 1995). An example is shown in Figure 3b. Such graphs are referred to as hysteresis curves and are known in other disciplines as phase space diagrams.

A way out of the multiple value dilemma regarding concentration and effect is known as the effect compartment model (Segre 1968, Verotta and Sheiner 1991). This approach postulates that the drug acts from a hypothetical effect compartment which uses an unobservable amount of drug. Concentration in the effect compartment, for cases considered in this paper, lags the concentration in the central blood compartment. By adjusting the elimination coefficient of the effect compartment (Keo), it is possible to "collapse the hysteresis loop" so that effect versus effect compartment concentration is a univalued function. Essentially the effect compartment concentration profile is shifted so that its time course coincides with the course of the effect.

In our study of data for nonsteroidal anti-inflammatory drugs (NSAIDs) given for pain and fever, we have used this effect compartment methodology to account for the data. However, delay mechanisms in such cases might arise from the existence of a classical feedback loop controlling the level of fever or pain (Katzper and Holford 1990). In the case of fever, the effect compartment approach is now not considered reasonable on physiological grounds (Roland and Tozer 1995). 
Later observations showed that open control loop mechanisms are sufficient to model many cases of interest including pain and fever (Dayneka et al. 1993, Katzper 1992). Jusko has disseminated this approach, calling it the indirect pharmacodynamic response model and applying it to a wide variety of drugs. In this model the effect is seen as the result of a balance of physiological forces, and the drug acts in affecting factors controlling the maintenance or loss of response. For this reason, it is referred to here as the dynamic response model.

The direct effect model, the cffect compartment model and the dynamic response model and their linkages are each illustrated schematically in Figure 1.

In subsequent sections these models will be explained further. Then by using identical pharmacokinetics, the pharmacodynamics of the models will be compared in a variety of physiological ranges.

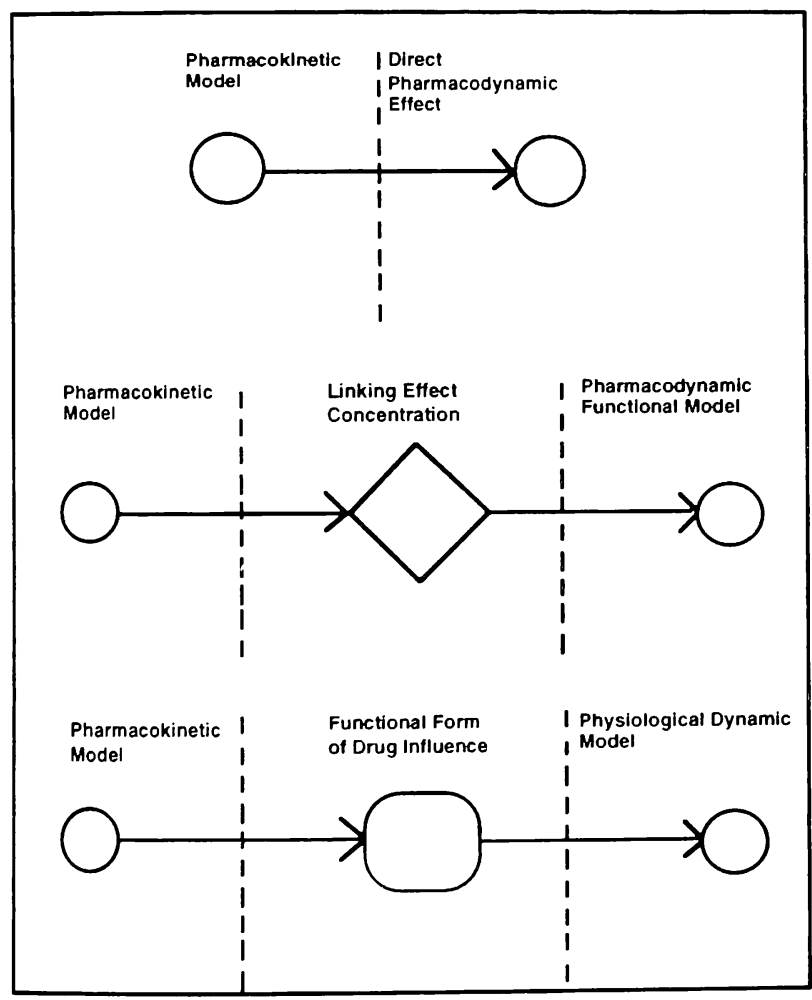

Figure 1: Pharmacokinetic/Pharmacodynamic Models

\section{PHARMACODYNAMIC FORMULATION}

Consider the pharmacodynamics of a one compartment model where the concentration in the blood is representative of the concentration in all affected parts of the body. A widely used empirical equation for relating concentrations directly to effects, and known by a number of different names, is the Emax model,

$$
\mathrm{E}(\mathrm{C})=\mathrm{E}_{0}+\mathrm{Emax}^{*} \mathrm{C} /(\mathrm{EC} 50+\mathrm{C})
$$

where $E_{0}$ is the baseline, Emax is the maximum difference from baseline, $\mathrm{C}$ is the concentration and EC50 is the level of concentration required to reach Emax/2 from the baseline. The effect depends on the current value of the concentration.

The equation captures the physiological reality of effects going to a limit. That is, the body can only respond up to a certain amount. At higher concentration values, one obtains smaller incremental responses. We use this equation in the different model constructs to be studied.

When multiple compartments are required for representing drug distribution and equilibration, and an effect depends on the concentration in a physiological compartment other than the central blood compartment, then there is a phase difference between the blood concentration profile and the effect profile. When such compartments are physiologically definable and they contain a non-negligible portion of the drug, then the influence of the additional compartments can be seen in the blood concentration profile. Visual inspection of blood concentration data thus provides partial guidance as to the choice of the appropriate compartmental model. When data is not available for the other compartments then the solution is frequently not unique, a difficulty referred to as the "identifiability problem."

Let us consider the case where drug response lags behind plasma concentration not due to an unequilibrated physiological compartment. If one is to subscribe to the notion that the effect is solely and instantaneously related to the drug concentration, then a possible explanation is in terms of an effect compartment which does not affect the blood level concentration noticeably. Specifically we will look at this model in the context of an intravenous infusion which is terminated after a time.

Our models are formulated using STELLA (Bogen 1989). The visual programming approach simplifies the formulation of complex models (Katzper 1992). Our model illustrations are presented using STELLA icons. A rectangle is a quantity or level. A pipe with a circle defines rates of change. Circles are for calculations or parameter settings. Arrows link quantities. For clarity of presentation some secondary calculations are suppressed in the STELLA model figures.

The top of Figure 2 shows a one compartment pharmacokinetic model with constant infusion, first order clearance and elimination rate $\mathrm{Ke}$. The middle of the figure is the effect compartment model. The line from Concentration to Transfer links the effect compartment to the plasma concentration. Keo is the dissipation rate for the effect compartment, which controlls the phase of this compartment with respect to the concentration. The Effect Concentration drives the Effect circle at the bottom of the figure. The Effect circle at the bottom contains the Emax equation. It is shown explicitly attached to it's parameters. 


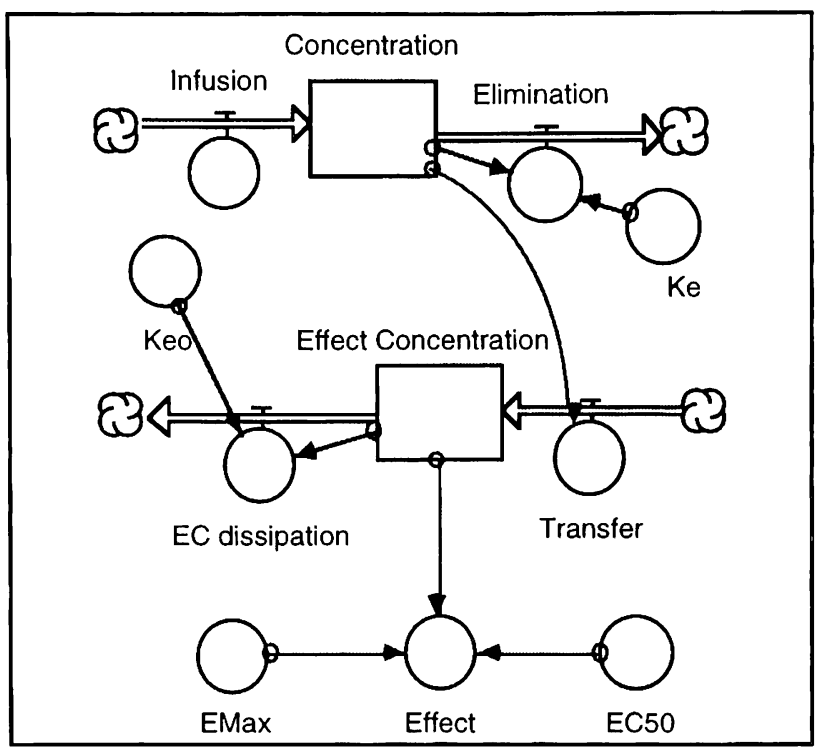

Figure 2: Concentration and Effect Compartment Model

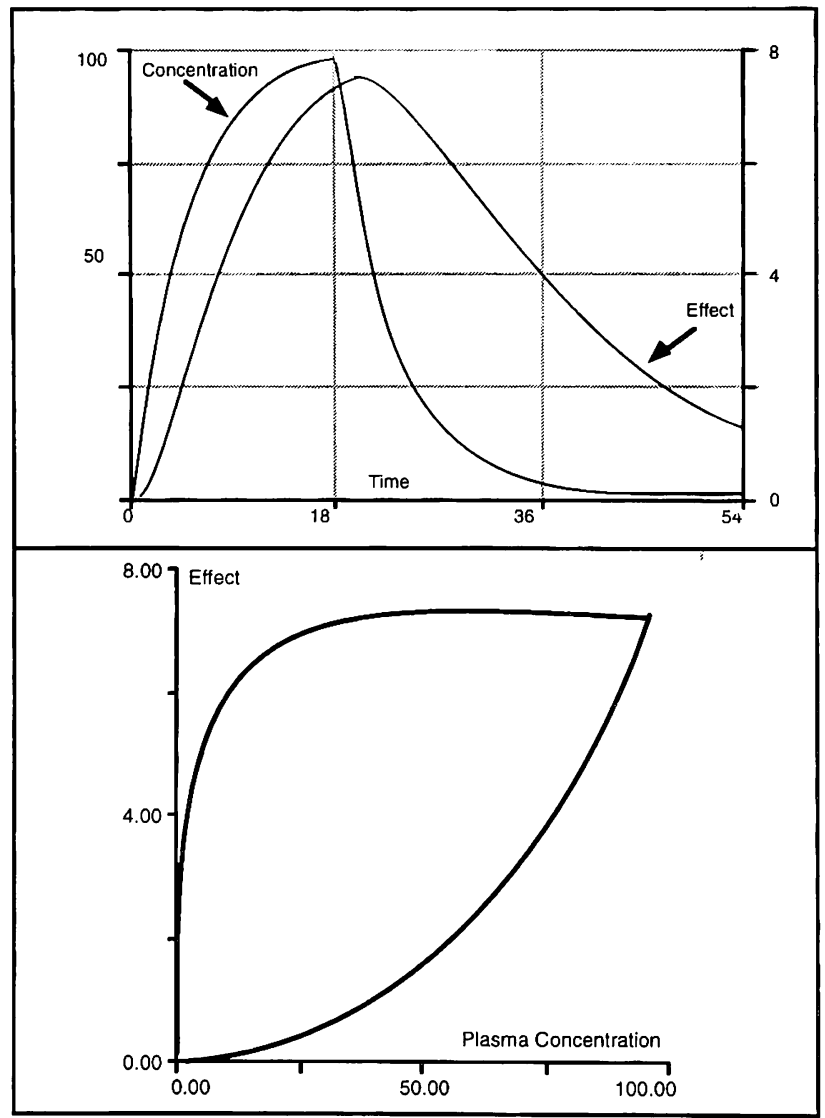

Figure 3a: Concentration and Effect time course 3b: Hysteresis loop. Effect versus Concentration

The plasma concentration of drug rises during the infusion and subsequently falls as shown for a sample case in Figure 3a together with the associated effect. Plotting effect versus plasma concentration, we obtain the hysteresis curve shown in Figure $3 b$.
The elimination coefficient of the effect compartment, $\mathrm{Keo}$, due to its controls of the effect compartment concentration with respect to the central compartment, controls the shape of the hysteresis curve. When experimental data is being fit, by varying $\mathrm{Keo}$, it is possible to "collapse the hysteresis loop" so that effect versus effect compartment concentration is a univalued function. This is achieved by alignment of the phase of the effect compartment concentration with the phase of the effect. In this way there is a one to one relationship between effect compartment concentration and the effect. In the simulation, Keo is specified and the effect compartment concentration drives the simultaneous Emax model.

If we consider the effect to be influencing a physiological dynamic process, then one way in which it can be modeled as an open loop control system. Such a control system can be represented as shown in the upper part of Figure 4. We shall refer to this model as the dynamic model as it attempts to represent the dynamics of the system. After a perturbation, the system will return to its equilibrium level. Under changed conditions, it will reequilibrate to a new level. The lower part of the figure is the drug pharmacokinetics. The connecting arrow between the models is a possible effect of the drug. The functional relationship is taken as an Emax form dependent on the plasma concentration, $\mathrm{C} /(\mathrm{C} 50+\mathrm{C})$ ,where $\mathrm{C} 50$ is the concentration to reach half maximal indirect influence on the effect. This equation is incorporated in the Link circle. The relationship can be thought of as a receptor response curve. Results similar to those shown in Figure 3 can be obtained for this model.

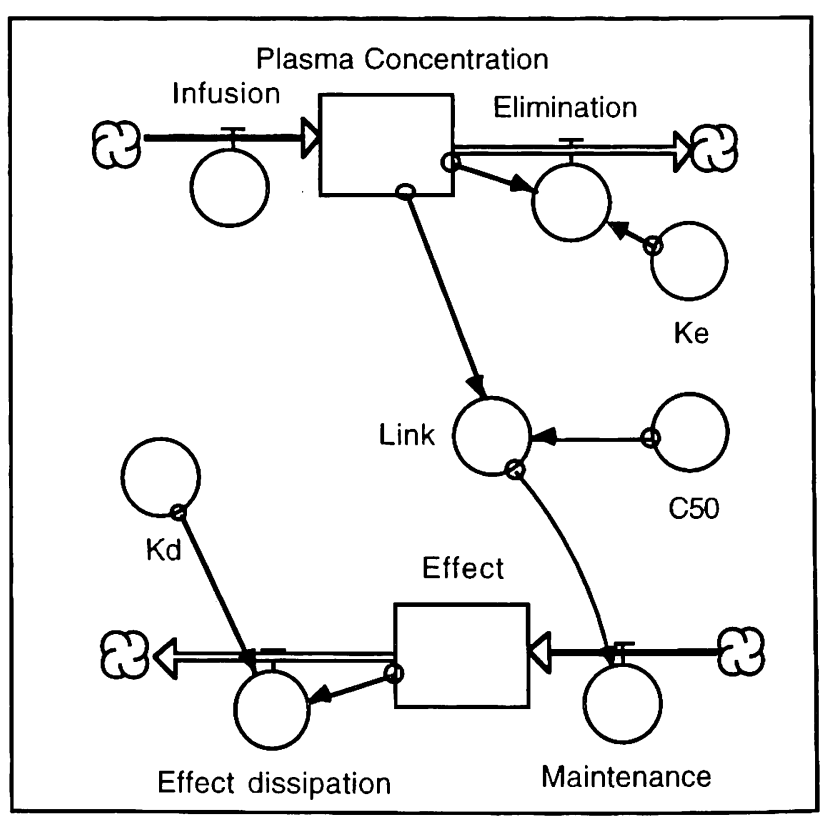

Figure 4: Dynamic Effect Model 


\section{COMPARATIVE DYNAMICS}

In their study of the dynamic response model, Dayneka et al. carried out a series of simulations comparing four variants of the model structure to the effect compartment model (Dayneka et al. 1993). Using literature values for the parameters of a selected drug (Methylprednisolone) they simulated widely differing dose levels with bolus or infusion delivery. They then investigated how well the effect compartment model could fit this data. Reasonable fits could be obtained for any given dose. The most telling critique was the observation of the dose dependency of the parameters to achieve the best fit. This is biologically implausible. Verotta turned the approach around (Verotta 1995). He showed, using simulation and experimental data, good fits with the effect compartment model in cases where the dynamic response model gave inferior results. None of this dose respone related work will be replicated here. Our conclusion from this dispute is that unbiased guidance is needed to assist in determining the appropriate model.

The question I thought worthwhile to explore was whether the models have distinctive intrinsic behavior which can be compared to experimental data to indicate which model to choose. When we look at the data that we collect for pain or fever alleviation, we seek to identify the underlying model which is the basis of the results we see. Identifications are strengthened if they match a distinctive feature in the time course of the data. To study the issue of intrinsic dynamic behavior, we chose parameters to represent distinctive clinical responses. Dosing was set to a constant level infusion for a fixed time. For such cases the concentration rises to a maximum and then decays, as shown in figure $3 a$, allowing us to observe the range of responses of the system. If the drug is effective, we expect pain or fever alleviation after a time lag, and as the concentration vanishes the pain or fever will resume if underlying conditions are unchanged. A number of reasonable models can fit this behavior. We therefore will look at our models over a range of parametric values to see if we can determine when the models results can be differentiated and when hysteresis representations help in the process of discrimination.

All of the cases are setup with the same pharmacokinetics. A single compartment model is used. An infusion of 20 units per time interval is given for 18 intervals. Data is collected for 18 more intervals. Subsequently this was extended to a total of 50 intervals to capture late differences between the models. The number of intervals affects the parameter estimates and the apparent closeness of the model results.

Elimination is via first order kinetics and the elimination constant is .2 per unit time. If the infusion were given till equilibration the concentration reached would be 100 units.

Since both our dynamic response model and the effect of the effect compartment model will be driven by Emax formulations the relative value of the relevant half maximum concentration (C50 or EC50, see Figure 2 and Figure 4) will affect the time course of the drug effect. So these two parameters should have a large degree of comparability. For convenience, in both cases we have set the baseline value $E_{0}$ to zero. For the effect compartment model the dynamics of concentration buildup in the effect compartment and the controller of phase is the elimination rate Keo. For increasing values of Keo the phase of the effect compartment will approach that of the central compartment. For the dynamic response model the speed of dissipation of prior infliences is controlled by $\mathrm{KD}$, the dissipation rate. Larger values of KD will have the effect more closely track the concentration. So we expect the effects of Keo and $\mathrm{KD}$ to be comparable. Experimentation with the models for different parametric values showed that this was the case. We present and discuss a number of examples systematically to show the relationship between parametric values and comparative model results. Matching of the models was carried out by fixing the parameters of one model and varying those of the second model. At first matching was done visually and then with a least squares fit.

Our first case is set for a large time lag between concentration and effect. As explained, this is achieved by setting $\mathrm{KD}$ and Keo low. The relationship between concentration and effect is set to be highly nonlinear. This is achieved by setting C50 and EC50 low with respect the associated concentrations achieved. The figures provide alternate representations comparing model results. Figure 5 shows a direct comparison of effect versus time.

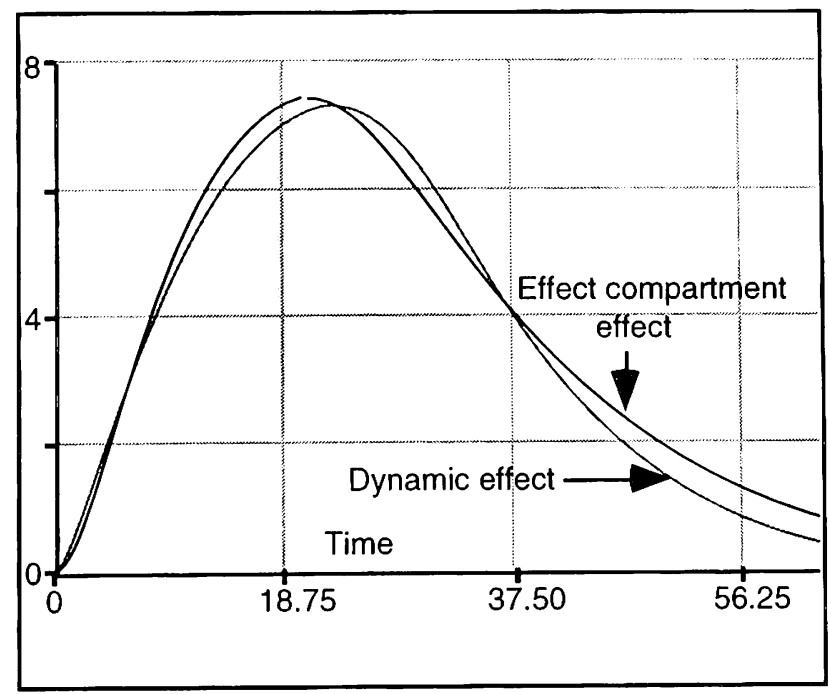

Figure 5: Comparative time course of effect 
Figure 6 is the hysteresis loop comparison. Direct comparison shows an apparent closeness in results between the two models, but with systematic differences. The large area enclosed by the hysteresis loop indicates a large lag in effect relative to plasma concentration.

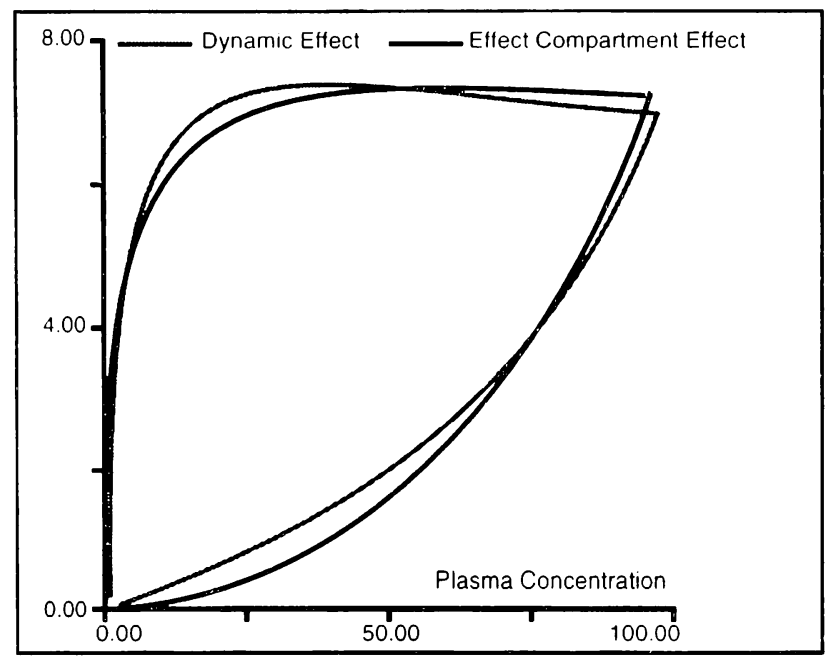

Figure 6: Comparative hysteresis loops

An interesting feature is that the dynamic model achieves its maximum fairly late during the decline of the concentration. Another feature, which is good from a medical perspective is the maintenance of the effect after infusion stops for a steep drop in concentration.

Figure 7, a plot of the effects predicted by one model versus the other, would give a straight diagonal line for identical models. As such, the deviations from fourty five degrees show the deviations of the models from each

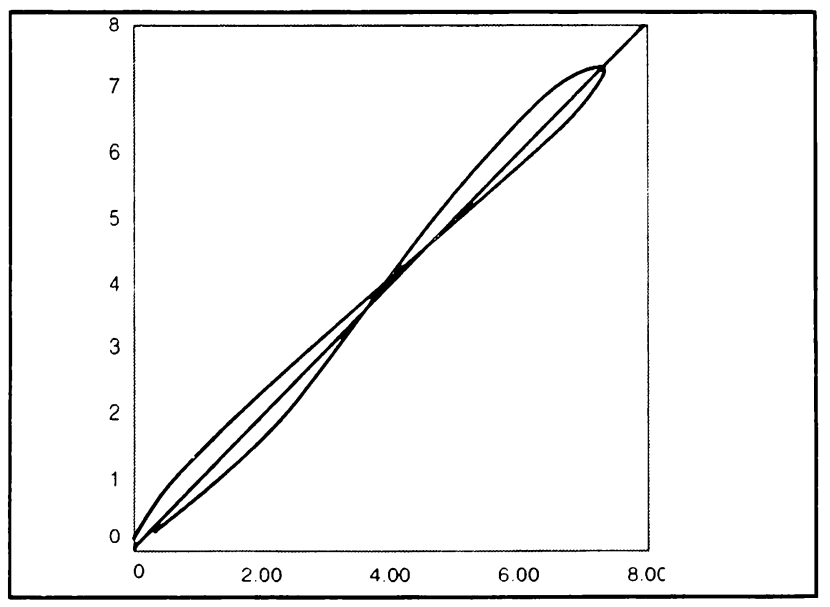

Figure 7: Comparative effect predictions

other. The reclining figure eight in this figure indicates the lead, lag, lead, lag, reversals of the two models. Now that we know this, by looking back at the other two representations, this pattern of lead and lag is also seen in those plots. Such systematic variation over a range of values, if it is sufficiently large, allows one to discriminate between models for a given set of data.

Figure 8 plots the difference between the models (Ec effect-dynamic effect) as a function of time. At this scale, the systematic variation in leading and lagging is very clear. The influence of the infusion and subsequent decay is noticeable.

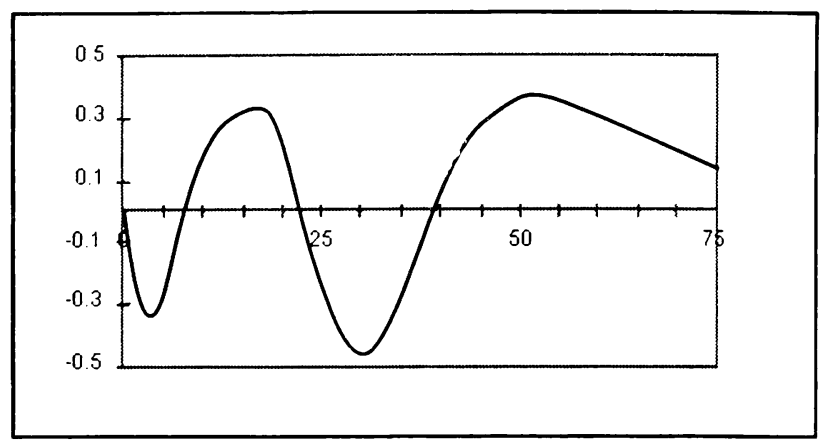

Figure 8: Time course of model difference

The second case was set to retain a large lag by keeping $\mathrm{KD}$ and Keo low but was set to approximate linearity in the dynamic model link by setting $\mathrm{C} 50$ high and in the effect link by setting EC50 high. One might think of the modeling process as the application of transformation operators on the input variables. An examination of the structure of the two models shows that they can be looked upon as two similar transformations taken in different sequence. The operations are non-commutative. However, for certain parametric combinations they approach commutativity. In plain English, the models approach each other in terms of their results.

For this case, we describe the graphic results. There is a near superposition of model results in a direct comparison versus time. The size of the effect is a little less than half of that in the first case. The hysteresis curve still has a large lag, but the linearity of the underlying relationship causes a slower rise in effect and a more rapid decline. This is the case for both models which are nearly superimposed. The direct comparison of the effects (not shown) maintains the reclining figure eight, but in a squashed form approximating a diagonal line. The models difference curve (not shown) retains lead lag variations because the plot scale is an order of magnitude smaller than the prior case. This demonstrates that systematic variation between the models is maintained. An improved match between the two models can not be expected from further parameter variation. This is acceptable for practical purposes as there is no known outcome which would be affected.

The third case is that of a small lag between blood concentration and effect. This is achieved by setting $\mathrm{KD}$ and Keo high. In addition the effect is in the linear relationship range for linking effect to concentration. Linearity is achieved by setting C50 and EC50 to high values. 
In this case, not only do the time curves of the models coincide but the effect curve closely approximates the shape of the infusion plasma concentration curve. At the selected parameter settings, both models still retain a lag as seen by the reduced enclosed area in Figure 9. The hysteresis curve for each model nearly coincide, though the minor differences are seen when high resolution graphics are used. A single example is shown here.

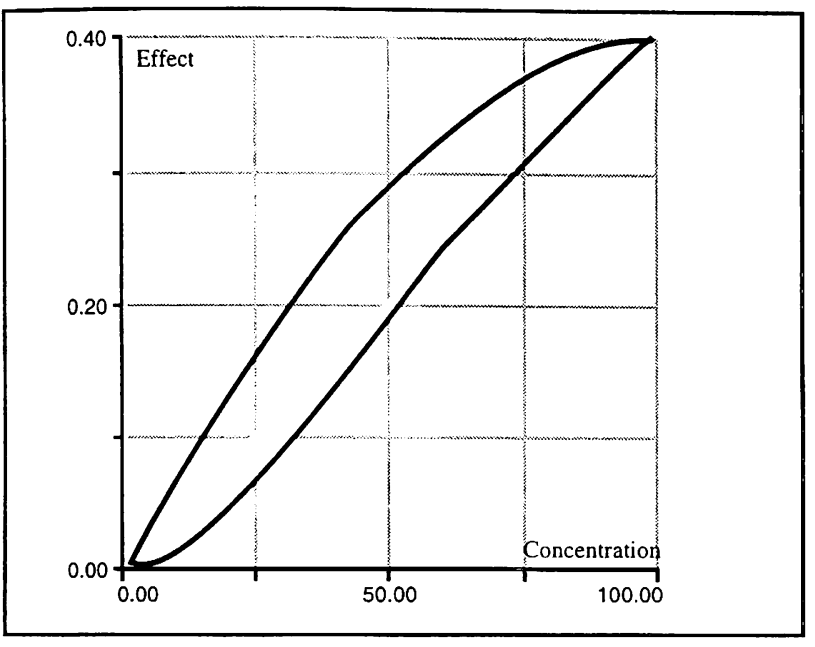

Figure 9: Effect versus concentration with reduced lag and a partially linearized link.

As $\mathrm{KD}$ and $\mathrm{Keo}$ are increased, the loop collapse tends to a straight line. The theoretical difference between the models is much smaller than measurement noise. This same loop collapse, which results in both models, has a completely different interpretation in each model. Here we directly face the issue that two very different models can be so dynamically similar as to be indistinguishable.

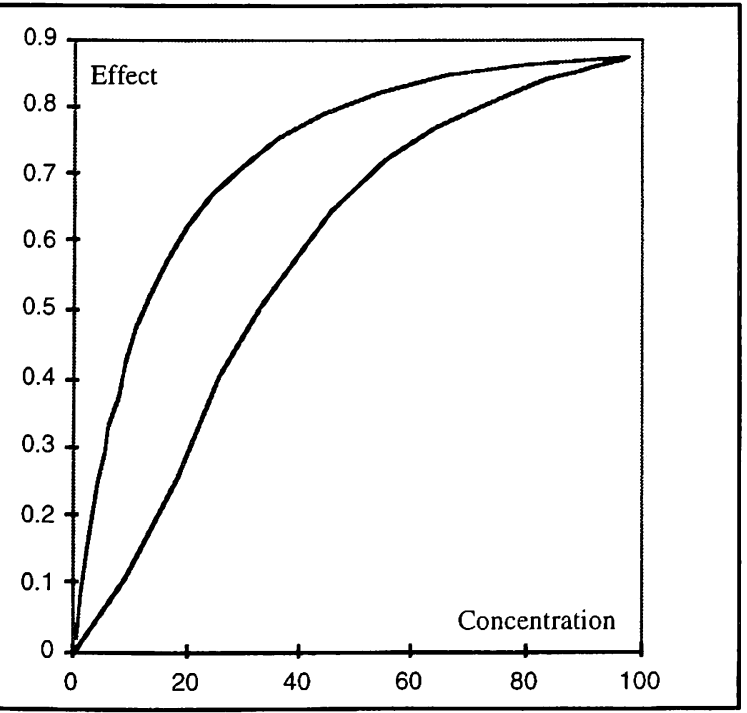

Figure 10: Consequence of a nonlinear link and reduced lag on the hysteresis curve
The final case in our illustrative set is also chosen to retain a small lag between blood concentration and effect. As before, the lag is achieved by keeping $\mathrm{KD}$ and $\mathrm{Keo}$ high. However, here the nonlinear range for linking effect to concentration has been chosen by setting C50 and EC50 lowwith respect to the concentration level achieved by the dosing regimen. The two parameters of the respective models can be adjusted so that the model results nearly coincide. The hysteresis curve, Figure 10, clearly shows the nonlinearity of the effect. The comparative computer graphics of the hysteresis curves (not shown here) demonstrate the retention of the lead-lag pattern, but with only a very small gap between the curves. The collapse of the area of the hysteresis curve in the limit will lead to a logistic like curve.

\section{DISCUSSION}

All of these studies have been made without the random variability which accompanies experimental data. A reason for this investigation was to determine whether the structural form of the model formulations was sufficient to create dynamic results which could not be made to coincide. Then it might be possible to differentiate between models based solely on the observed data even when noise is present. Furthermore, different graphical representations were used to enhance the difference signal. The rather unexpected result, as has been shown, is that for the present dosing regimen, differences are discernible only in the parameter range giving strong model effects of large lag and nonlinearity. The limits of differentiation are dependent on the noise level. Combining information regarding the intrinsic response with results from multiple dose level fitting will enhance model discrimination.

Real data also do not allow for the density of observations or the length of observation used here. It must be kept in mind that we have purposely dealt with an idealized case. In our studies of pain the data are more complex with contributions from placebo effects and gastric transit variability forcing us to construct more complex models (Harter and Katzper 1994).

In matching complex data our guidance has come from having multiple data sources and from physiological considerations (Harter and Katzper 1993, Harter and Katzper 1995).

Phase relationships between the models require a long interval of data for the changing relationships to aid in discerning which model is in better agreement with the data. Short interval fits will not do the job.

This model study with its limited focus only provides general guidance for the real problems we are addressing. Additional sources of information must be sought in deciding which model is more appropriate. 


\section{CONCLUSION}

To assist in interpreting the time course and hysteresis loops of the data we have seen, a series of simulation experiments have been carried out for competing models. A selected summary of these runs has been presented. These are used to compare results from matched alternate models. The simulations have shown under what conditions model results are most easily differentiated. Under single dose conditions, maximum separation will occur for the greatest lag between concentration and effect and for highly nonlinear links between the pharmacokinetic and pharmacodynamic model components.

A finding which is important to note is that in spite of disparate conceptual formulations, the intrinsic model dynamics is such that it is frequently difficult to discriminate between models. Parametric values of interest are physiologically determined. Current experimental approaches are not intentionally designed to affect these physiological values directly. Discrimination may be achieved by observation of many individuals with their naturally occurring physiological variability. This is especially so for drugs in which model effects are strong.

On the other hand, with only limited noisy data, one might be misled by the model fit obtained. When both models are approximately equivalent in their ability to fit available data one may ask why worry about which model is used? Because the model is our conceptual framework for understanding the subject matter. A model corresponding to the underlying reality has the possibility of forming the basis for further fruitful investigation and elaboration. In the converse situation, we have a well fitted dead end.

\section{REFERENCES}

Bogen, D. K. 1989. Simulation Software for the Macintosh. Science 246:138-142.

Dayneka, N. L., Garg, V., and W. J. Jusko 1993. Comparison of Four Basic Models of Indirect Pharmacodynamic Responses. Journal of Pharmacokinetics and Biopharmaceutics. 21:457-478.

Harter, J. G. and M. Katzper. 1993. Therapeutic Implications of Effector Response Alternatives. In Proceedings of the SCS Multiconference on Simulation in the Health Sciences and Services, 66-70. Society for Computer Simulation, San Diego, California.

Harter, J. G. and M. Katzper. 1994. Refinements in Modeling Pain and Its Response to Analgesics. Clinical Pharmacology and Therapeutics, 55:123.
Harter, J. G. and M. Katzper. 1995. Components of Pain Alleviation. Westerm Simulation Multiconference: Health Sciences, Physiological and Pharmacological Simulation Studies, 159-164. Society for Computer Simulation, Las Vegas, Nevada.

Katzper, M. and N. H. G. Holford. 1990. Alternate Pharmacodynamic Models for Fever Reduction by Ibuprofen. Third Symposium: Frontiers of Pharmacokinetics and Pharmacodynamics. Baltimore, Maryland.

Katzper, M. 1992a The Use of Visual Programming for Pharmacokinetic and Pharmacodynamic Simulation.

Proceedings of the SCS Multiconference on Simulation in Health Care and Social Services, 65-69. Society for Computer Simulation, Newport Beach, California.

Katzper, M. 1992b Control System Underpinning of Hysteresis Loops. Second Jerusalem Conference on Pharmaceutical Sciences and Clinical Pharmacology. Jerusalem, Israel.

Roland, M. and Tozer, T. N. 1995. Clinical Pharmacokinetics: Concepts and Applications. 3rd edition. Baltimore: Williams and Wilkins.

Segre, G. 1968. Kinetics of Interactions Between Drugs and Biological Systems. Il Farmaco, 23:907-918

Verotta, D. and L. B. Sheiner. 1991. Semiparametric Analysis of Non-Steady-State Pharmacodynamic Data." J. Pharmacokinetics and Biopharmaceutics, 19:691-712.

Verotta, D. 1995. Semiparametric Direct and Indirect Action Models for Pharmacokinetic/Pharmacodynamic Data. Western Simulation Multiconference: Health Sciences, Physiological and Pharmacological Simulation Studies. 177-182. Society for Computer Simulation, Las Vegas, Nevada.

\section{AUTHOR BIOGRAPHY}

MEYER KATZPER is a research analyst at the Center for Drug Evaluation and Research (CDER). He carries out modeling and analytic studies to assist in the drug review process. He teaches introductory and advanced modeling courses in the CDER Staff College. 